Check for updates

Cite this: RSC Adv., 2018, 8, 34314

Received 7th August 2018

Accepted 1st October 2018

DOI: $10.1039 / c 8 r a 06639 f$

rsc.li/rsc-advances

\section{Two-step photomechanical motion of a dibenzobarrelene crystal $\dagger$}

\author{
Takuya Taniguchi, (D) a Ayumi Kubota, ${ }^{\text {b }}$ Tatsuya Moritoki, ${ }^{\mathrm{c}}$ Toru Asahi $^{\mathrm{ad}}$ \\ and Hideko Koshima (iD) *d
}

Photomechanical crystals are interesting from both basic and applied perspectives, and thus it is important to develop new examples. We investigated the photomechanical bending behaviour of a photochromic crystal of a dibenzobarrelene derivative. When a plate-like crystal was irradiated with ultraviolet (UV) light at $365 \mathrm{~nm}$, two-step bending was observed. In the first step, the crystal quickly bent away from the light source, with an accompanying crystal colour change from colourless to purple. In the second step, under prolonged UV light, the bending returned slowly and then the crystal bent up towards the opposite direction, accompanied by an additional colour change to light yellow. Spectroscopic measurements and X-ray crystallographic analysis suggested that a long-lived biradical species is generated immediately upon UV light irradiation via a Norrish type II intramolecular hydrogen abstraction, and then the final photoproducts are formed under continuous UV exposure. X-ray crystallographic analysis before and after UV light irradiation for a few seconds revealed that the longitudinal axis (a axis) of the crystal elongated slightly after irradiation, which is consistent with the direction of the first-step bending. Based on these results, we propose that first-step bending could be induced by a biradical species, generated via a Norrish type II intramolecular hydrogen abstraction, and the second-step bending could originate from the formation of a mixture of final photoproducts under prolonged light irradiation.

\section{Introduction}

Materials that move by responding to external stimuli, such as light, heat, electricity, magnetic field, pressure, etc., have attracted substantial attention in recent years. Such mechanically responsive materials are expected to find applications as actuators and sensors for soft robots. Photomechanical polymers and gels have been well developed in previous research., ${ }^{\mathbf{1 , 2}}$

Contrary to their appearance as solid but fragile objects, in the past decade molecular crystals have been attractive because they exhibit mechanical motion induced by external stimuli. Typical photochemical reactions, such as pericyclic reactions, ${ }^{\mathbf{3}, 4}$ trans-cis isomerisation, ${ }^{\mathbf{5 , 6}}$ enol-keto isomerisation, ${ }^{7}[2+2]$

\footnotetext{
${ }^{a}$ Department of Advanced Science and Engineering, Graduate School of Advanced Science and Engineering, Waseda University, 3-4-1 Okubo, Shinjuku-ku, Tokyo 169-8555, Japan

${ }^{b}$ Department of Life Science and Medical Bioscience, School of Advanced Science and Engineering, Waseda University, 3-4-1 Okubo, Shinjuku-ku, Tokyo 169-8555, Japan 'Department of Materials Science and Biotechnology, Graduate School of Science and Engineering, Ehime University, 3 Bunkyo-cho, Matsuyama, Ehime 790-8577, Japan ${ }^{d}$ Research Organization for Nano \& Life Innovation, Waseda University, 513 Wasedatsurumaki-cho, Shinjuku-ku, Tokyo 162-0041, Japan. E-mail: hkoshima@ aoni.waseda.jp

$\dagger$ Electronic supplementary information (ESI) available. CCDC 1860420-1860422. For ESI and crystallographic data in CIF or other electronic format see DOI: 10.1039/c8ra06639f
}

cycloaddition, ${ }^{\mathbf{8} 9}$ and $[4+4]$ dimerisation, ${ }^{\mathbf{1 0 - 1 2}}$ induce various kinds of photomechanical motion in molecular crystals. Recent advances in photomechanical crystals have been summarised in several reviews. ${ }^{\mathbf{1 3 - 1 8}}$ Although many photomechanical crystals have been developed so far, the types of photochemical reactions that have been used to achieve photo-induced motion are limited. Thus, there is a need to explore other photochemical reaction systems to evaluate a wider variety of mechanical behaviour.

Dibenzobarrelene derivatives are well known to partake in various photochemical reactions in solution, such as tri- $\pi$ methane rearrangement, di- $\pi$-methane rearrangement, Norrish type II reactions, and $[2+2]$ cycloaddition. ${ }^{19-21}$ The solid state photochemistry of dibenzobarrelenes has also been wellstudied, by Scheffer and co-workers. ${ }^{22,23}$ Photochromism in the solid state of a dibenzobarrelene derivative was reported in detail by Ramaiah and co-workers, in which the photochromism is caused by a long-lived triplet biradical, formed from a Norrish type II intramolecular hydrogen abstraction initiated by ultraviolet (UV) light irradiation. ${ }^{24}$ However, photochromism in other dibenzobarrelene derivatives has not been wellstudied.

In this study, we investigated the photochromism and bending behaviour of a similar dibenzobarrelene derivative, namely, 11,12-dibenzoyl-9,10-dihydro-9,10-dimethyl-9,10- 
ethenoanthracene (1), which was reported to exhibit a colour change from colourless to purple after UV light irradiation (Fig. 1). ${ }^{24}$ In terms of photomechanical motion, the plate-like crystal of compound 1 was observed to bend quickly with the colour change (colourless to purple) initiated by UV light irradiation. Following this, the bent crystal slowly bent up towards the opposite direction, with additional colour changes from purple to yellow under prolonged UV light exposure. The mechanism of the two-step photomechanical bending motion of the compound $\mathbf{1}$ crystal is discussed with respect to spectral and crystal structure changes.

\section{Experimental}

\section{Materials}

Compound 1 was synthesised according to the same scheme used in previous reports. ${ }^{19,25}$ Compound 1 was purified by silica gel column chromatography (hexane : ethyl acetate $=10: 1$ ). After purification, plate-like crystals were obtained by evaporation of the solvent mixture. The compound was identified by nuclear magnetic resonance (NMR) and melting point measurements. The melting point was determined with the MPS3 Micro Melting Point System (Yanako New Science, Inc.).

\section{Diffuse reflectance measurements}

UV-Vis diffuse reflectance spectra of a powdered sample of compound 1 were measured with a Lambda 650 spectrometer (PerkinElmer). The powder was mounted on a glass plate and then covered with a quartz glass plate. In each experiment, the sample was picked up from the measurement holder, and then irradiated by UV light $\left(365 \mathrm{~nm}, 40 \mathrm{~mW} \mathrm{~cm}^{-2}\right.$ ) from the quartz glass side. Every measurement was performed in the range 200$800 \mathrm{~nm}$ at $1 \mathrm{~nm}$ resolution with a scan speed of $200 \mathrm{~nm} \mathrm{~min}{ }^{-1}$.

\section{FT-IR measurements}

Fourier-transform infrared (FT-IR) spectra of a powdered sample of compound 1 were measured with an attenuated total reflection (ATR) method using a Nicolet 6700 spectrometer (Thermo Fisher Scientific) in the range $500-4500 \mathrm{~cm}^{-1}$. The sample was irradiated by UV light $\left(365 \mathrm{~nm}, 40 \mathrm{~mW} \mathrm{~cm}^{-2}\right)$.
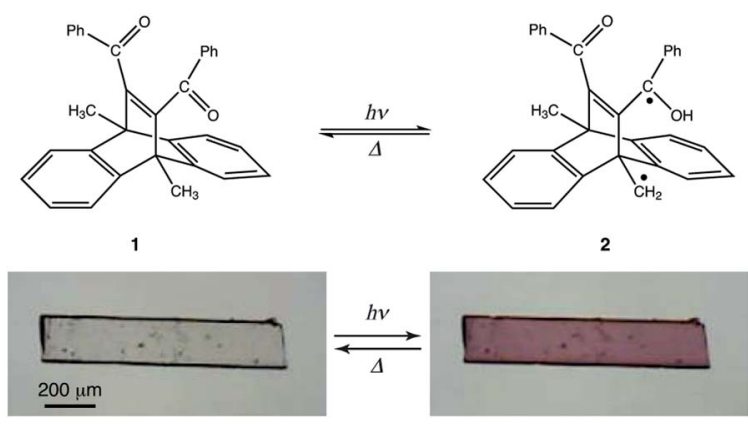

Fig. 1 Photochromism of dibenzobarrelene 1 in the solid state.

\section{X-ray crystallographic analysis}

Single-crystal diffraction data for compound $\mathbf{1}$ were collected using an R-AXIS RAPID diffractometer (Rigaku) equipped with monochromatic $\mathrm{Cu}-\mathrm{K} \alpha$ and $\mathrm{Mo}-\mathrm{K} \alpha$ radiation sources. Measurements were performed at 223 and $293 \mathrm{~K}$. The low temperature measurements were conducted to obtain the crystal structure after light irradiation. At first, measurements were taken before irradiation at $223 \mathrm{~K}$. Following this, the crystal sample was irradiated with UV light for several seconds at $293 \mathrm{~K}$ while being rotated, as photochemical reactions are slow at low temperature. After irradiation, the structure was determined again at $223 \mathrm{~K}$ to preserve photoproducts in the crystal. The crystal structure at $293 \mathrm{~K}$ in the absence of UV light was also determined, to correlate with the bending behaviour observed at room temperature. Crystal structures were solved by using the direct method of SHELXD2013, and refined on $F^{2}$ by the full-matrix least-squares method, SHELXL. ${ }^{26}$ Calculations were performed using the software package CrystalStructure (Rigaku). ${ }^{27}$

\section{Observation of bending behaviour}

Following flash column chromatographic purification, thin plate-like crystals were obtained by solvent evaporation. A crystal was fixed at the tip of a glass needle with glue, and then irradiated by $365 \mathrm{~nm}$ UV light with a flux density of $40 \mathrm{~mW} \mathrm{~cm}^{-2}$ (UV-400; Keyence). Movies were recorded using a digital highspeed microscope (Keyence; VHX-5000).

\section{Results and discussion}

\section{Diffuse reflectance spectra}

Diffuse reflectance spectra of a powdered sample of compound 1 were measured. Prior to UV light irradiation, the spectrum had a broad peak between 200-400 nm, and no peaks above $450 \mathrm{~nm}$ (Fig. 2a). Upon UV light irradiation for $0.1 \mathrm{~s}$, a peak appeared at $574 \mathrm{~nm}$, for which the peak intensity increased with irradiation time until $3 \mathrm{~s}$, with a corresponding colour change from virtually colourless to purple (Fig. 2a). Difference spectra, obtained by subtracting the spectrum before UV light irradiation from each spectrum after light irradiation, highlight these spectral changes (Fig. 2b). The difference spectra exhibit three peaks at 340, 430, and $574 \mathrm{~nm}$, all of which increased with irradiation time (Fig. 2b). The temporal behaviour of the peaks at 340 and $430 \mathrm{~nm}$ were similar, but in contrast to that of $574 \mathrm{~nm}$ (Fig. S1a $\dagger$ ). The largest increase in intensity was for the $574 \mathrm{~nm}$ peak, which indicates the generation of a purplecoloured species, consistent with the colour of the powder after irradiation (Fig. S2a $\dagger$ ).

The absorption peak at $574 \mathrm{~nm}$ can be attributed to the extensive $\pi$-delocalisation of the biradical species between the triplet benzyl radical and the benzoyl groups, which are connected by the conjugated alkenyl double bond. ${ }^{24}$ The increase in peak intensity at $574 \mathrm{~nm}$ until $3 \mathrm{~s}$ after irradiation suggests that biradical 2 was produced immediately by a Norrish type II intramolecular hydrogen abstraction. The apparent half-life $\left(\tau_{1 / 2 \mathrm{UV}}\right)$ of hydrogen abstraction from compound $\mathbf{1}$ to generate 
(a)

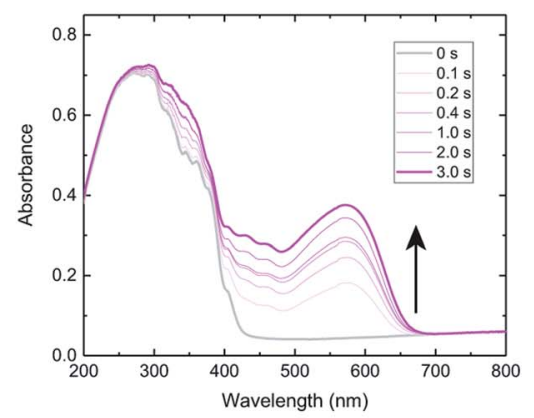

(b)

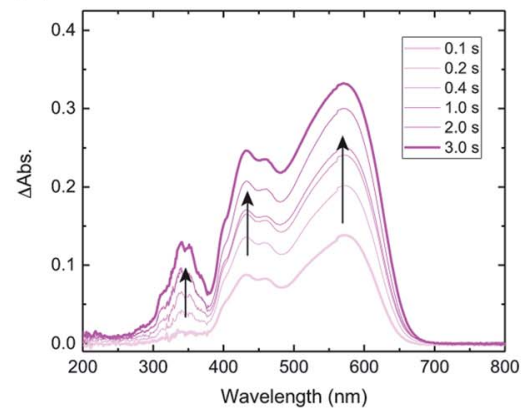

(c)

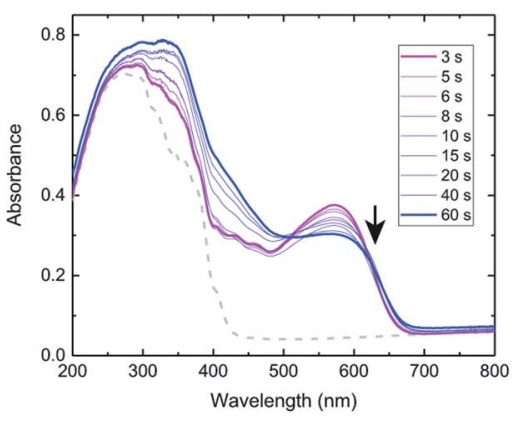

(d)

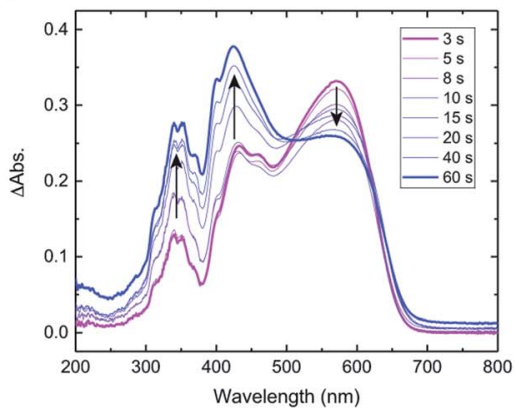

(e)

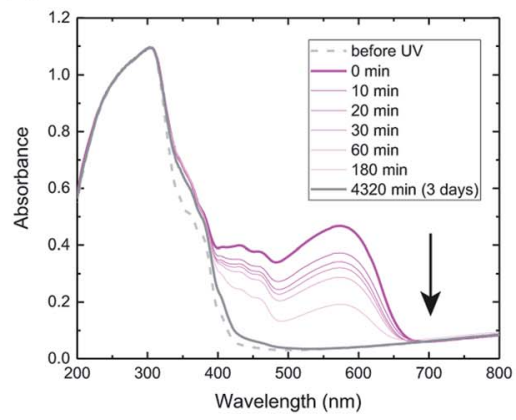

(f)

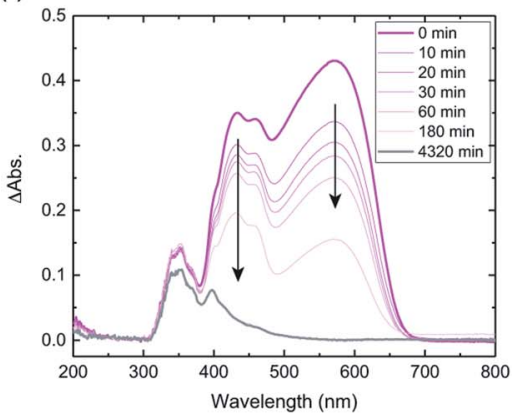

Fig. 2 (a, c and e) Diffuse reflectance spectra and (b, $d$ and f) difference spectra of a powdered sample of compound 1. (a-d) Changes induced by ultraviolet (UV) light irradiation; ( $a$ and b) in the initial $3 \mathrm{~s}$, and (c and d) for $60 \mathrm{~s}$ of UV exposure. (e and f) Thermal relaxation after UV light exposure for $3 \mathrm{~s}$. Black arrows indicate changes in peak intensities. Difference spectra were obtained by subtracting the spectrum before UV light irradiation from spectra obtained under or after irradiation.

biradical 2 was estimated to be $0.12 \mathrm{~s}$, based on the initial changes in peak intensity at $574 \mathrm{~nm}$ (Fig. S1b†). The peak at $430 \mathrm{~nm}$ can also be assigned to biradical 2, as reported previously. ${ }^{24,28,29}$

After stopping UV light irradiation for $3 \mathrm{~s}$, the absorption peak at $574 \mathrm{~nm}$ decreased gradually with time, and its initial intensity returned completely within 3 days (Fig. 2e). However, the difference spectrum after 3 days indicates that spectral changes for the peaks at 340 and $400 \mathrm{~nm}$ clearly remained (Fig. 2f), especially at $340 \mathrm{~nm}$, for which virtually no decrease was observed (Fig. S1g and $h \dagger$ ); this suggests that final photoproducts were produced. This spectral behaviour indicates that biradical 2 largely reverted to initial compound $\mathbf{1}$, and that the remaining absorption peaks after 3 days should be assigned to $\pi$-conjugated final photoproducts produced by irreversible tri$\pi$-methane and di- $\pi$-methane rearrangements, which are reported to occur in benzene solution (Fig. S3†). ${ }^{20}$ The photoproducts via the rearrangements in the crystals should be produced immediately upon UV irradiation based on the absorption increase at $340 \mathrm{~nm}$ by light irradiation (Fig. 2b). The half-life of thermal relaxation $\left(\tau_{1 / 2}\right)$ from biradical 2 into compound 1 was estimated to be $30.6 \mathrm{~min}$, based on the initial changes in peak intensity at $574 \mathrm{~nm}$ (Fig. S1c and $\mathrm{d} \dagger$ ); this is almost coincident with that $\left(\tau_{1 / 2}=39.2 \mathrm{~min}\right)$ estimated at $430 \mathrm{~nm}$ (Fig. S1e and f $\dagger$ ). The slow thermal relaxation of biradical 2 originates from the long lifetime of the triplet state biradical.

When the sample was irradiated for longer than $3 \mathrm{~s}$, the peak intensity at $574 \mathrm{~nm}$ decreased gradually, but the intensities at
340 and $430 \mathrm{~nm}$ continued to increase with irradiation time (Fig. 2c). Difference spectra show these changes in the three peaks clearly (Fig. 2d). After irradiation for $60 \mathrm{~s}$, the intensity at $430 \mathrm{~nm}$ increased the most, which is consistent with the observations of yellow-coloured material in the powder (Fig. S2b†).

The gradual decrease in the peak at $574 \mathrm{~nm}$ under prolonged UV light exposure might be due to the coupling of biradical 2 to produce cyclobutanol, which prohibits radical delocalisation (Fig. S3†). In terms of the photochemistry of benzophenone derivatives in the solid state, such cyclobutanol formation from biradical species has been well-documented. ${ }^{30-32}$ The continuous increase in the peak at $430 \mathrm{~nm}$ indicates that a biradical species is produced under light, but that the biradical cannot delocalise; this may be due to cyclisation. The continuous increase in the peak at $340 \mathrm{~nm}$ suggests that photoproducts of di- $\pi$-methane and tri- $\pi$-methane rearrangements are also continuously produced. Therefore, after prolonged light irradiation, the powdered sample could be a mixture of the initial compound, biradical 2, cyclobutanol, and the photoproducts from rearrangements (Fig. S3†).

\section{FT-IR spectra}

FT-IR spectra of a powdered sample of compound 1 were measured after UV light irradiation (Fig. 3). Before light irradiation, the spectrum had two peaks in the range 1600$1700 \mathrm{~cm}^{-1}$, at 1649 and $1664 \mathrm{~cm}^{-1}$, which were attributed to the $\mathrm{C}=\mathrm{O}$ stretching vibrations of compound 1 . These two peaks 


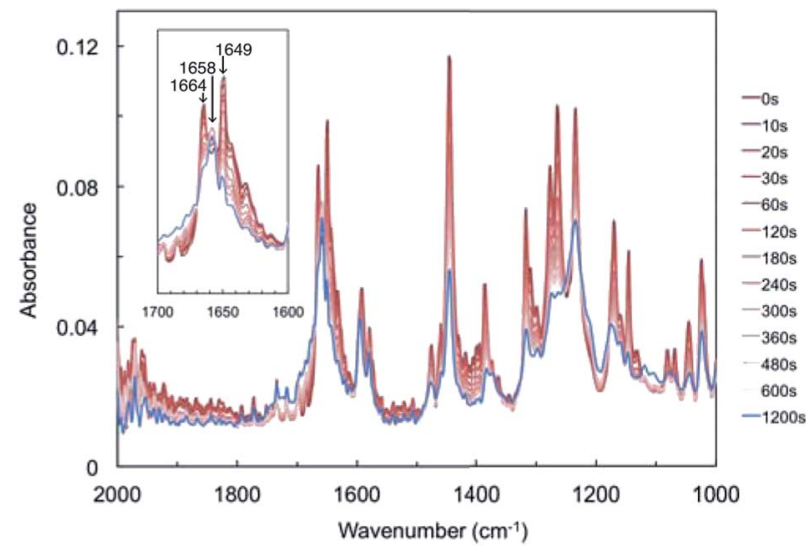

Fig. 3 Dependence of Fourier-transform infrared (FT-IR) spectral changes on UV irradiation time. The inset shows enlarged spectra in the range of $1600-1700 \mathrm{~cm}^{-1}$. The peaks at 1649 and $1664 \mathrm{~cm}^{-1}$ can be attributed to the $\mathrm{C}=\mathrm{O}$ stretching vibration of compound 1 , and the peak at $1658 \mathrm{~cm}^{-1}$ might be attributed to biradical 2, generated from hydrogen abstraction.

decreased with increasing irradiation time, and a new peak appeared at $1658 \mathrm{~cm}^{-1}$, which increased upon prolonged UV light exposure. The temporal behaviour of these three peaks is summarised in Fig. S4. $\dagger$ The two peaks at 1649 and $1664 \mathrm{~cm}^{-1}$ continued to decrease, while the new peak at $1658 \mathrm{~cm}^{-1}$ increased for $60 \mathrm{~s}$ and then reached saturation (Fig. S4a and $\mathrm{b} \dagger$ ).

The new peak at $1658 \mathrm{~cm}^{-1}$ might be attributed to biradical species 2. Here, the $\mathrm{C}=\mathrm{O}$ stretching vibrations of cyclobutanol and rearrangement photoproducts were not detected, probably due to low conversion rates or spectral overlap with the peaks of compound 1. These changes suggest that a Norrish type II intramolecular $\gamma$-hydrogen abstraction proceeded to decrease the $\mathrm{C}=\mathrm{O}$ bond strength of compound $\mathbf{1}$ to form biradical 2 .

\section{Crystal structure}

X-ray crystallographic analysis was performed at $293 \mathrm{~K}$ (Fig. 4 and Table S1 $\dagger$ ). The ORTEP drawing of compound $\mathbf{1}$ is shown by thermal ellipsoids with $25 \%$ probability (Fig. 4 a). The molecule has a disordered phenyl ring with an occupancy of $56 \%$ as its main conformation (Fig. 4a).

To analyse the intramolecular hydrogen abstraction reaction, we used four geometric parameters, $d, \Delta, \theta$, and $\omega$ (Fig. 4b), which were first introduced by Scheffer for the analysis of $\gamma$ hydrogen abstractions in the solid state..$^{33}$ These parameters are defined as follows: $d$ is the distance between the carbonyl oxygen and the $\gamma$-hydrogen atom, $\Delta$ is the $\mathrm{C}=\mathrm{O} \cdots \mathrm{H}$ angle, $\theta$ is the $\mathrm{C}-\mathrm{H} \cdots \mathrm{O}$ angle, and $\omega$ is the angle by which the $\gamma$-hydrogen atom lies outside the mean plane of the carbonyl group (Fig. 4b). The previous paper reported that the favourable $d$ is the sum of the van der Waals radii of hydrogen and oxygen atoms $(2.72 \AA)$, and that favourable angle geometries of $\omega, \Delta$, and $\theta$ are around $50^{\circ}, 80^{\circ}$, and $115^{\circ}$, respectively. ${ }^{33}$

In Table 1, these parameters are summarised for compound 1. Here, only H1A and H2A (shown in Fig. 4a) were considered in the $\mathrm{C}=\mathrm{O} \cdots \mathrm{H}$ geometry, because the other methyl group hydrogen atoms are too far away from the carbonyl $\mathrm{O} 1$ and $\mathrm{O} 2$

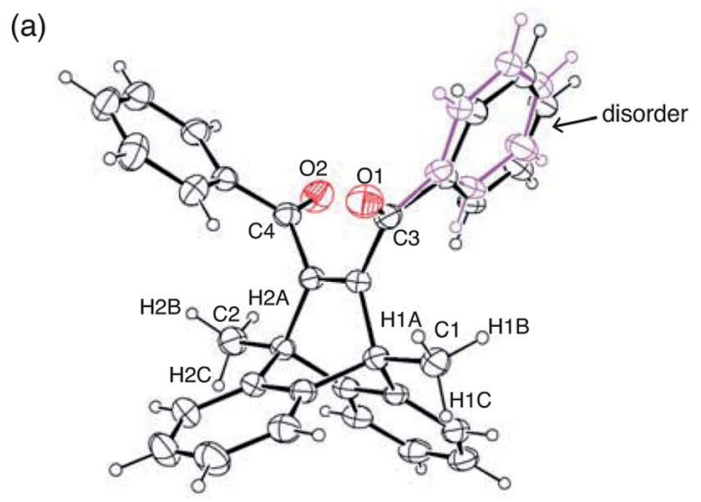

(b)
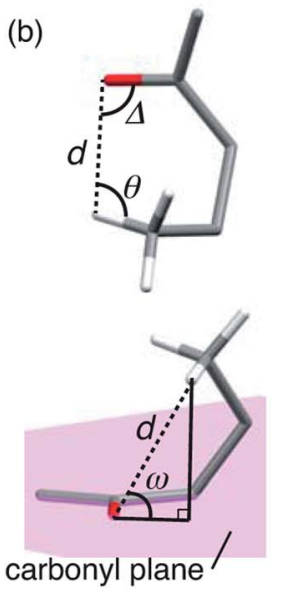

Fig. 4 Crystal structure of compound 1 at 293 K. (a) Molecular conformation drawn by thermal ellipsoids with $25 \%$ probability. The main conformation of the disordered phenyl ring is drawn in black (the same colour as other carbons), and the minor conformation is drawn in purple. (b) Definition of geometric parameters for $\gamma$-hydrogen atom abstraction: $d$ is the distance between the carbonyl oxygen and the $\gamma$ hydrogen, $\Delta$ is the $\mathrm{C}=\mathrm{O} \cdots \mathrm{H}$ angle, $\theta$ is the $\mathrm{C}-\mathrm{H} \cdots \mathrm{O}$ angle, and $\omega$ is the angle by which the $\gamma$-hydrogen atom lies outside the mean plane of the carbonyl group. (c) Molecular arrangement of the (001) face, which is the largest face of the plate-like crystal of compound 1. Hydrogen atoms and the disordered phenyl ring are omitted for clarity.

atoms. The distances $d$ of $\mathrm{C} 1=\mathrm{O} 1 \cdots \mathrm{H} 1 \mathrm{~A}$ and $\mathrm{C} 2=\mathrm{O} 2 \cdots \mathrm{H} 2 \mathrm{~A}$ are 2.656 and $2.874 \AA$, respectively, both of which are close to the favourable distance $(2.72 \AA)$. In terms of angle requirements, the values of $\omega, \Delta, \theta$ were $59.4^{\circ}, 83.8^{\circ}$, and $111.3^{\circ}$ for $\mathrm{C} 1=\mathrm{O} 1 \cdots$ $\mathrm{H} 1 \mathrm{~A}$, and $63.6^{\circ}, 72.7^{\circ}$, and $110.7^{\circ}$ for $\mathrm{C} 2=\mathrm{O} 2 \cdots \mathrm{H} 2 \mathrm{~A}$, respectively. These angles are also similar to the favourable geometries. Based on this analysis, it is estimated that both carbonyl groups can abstract hydrogen atoms from methyl groups to produce biradical 2. The biradical species may cause further radical coupling, because the distances of both $\mathrm{C} 1 \cdots \mathrm{C} 3(2.92 \AA)$ and $\mathrm{C} 2 \cdots \mathrm{C} 4(2.88 \AA)$ allow for the formation of cyclobutanol, as reported in benzophenone crystals. ${ }^{30-32}$

Table 1 Geometric parameters for hydrogen abstractions

\begin{tabular}{lllll}
\hline & $d(\AA)$ & $\omega\left(^{\circ}\right)$ & $\Delta\left(^{\circ}\right)$ & $\theta\left({ }^{\circ}\right)$ \\
\hline $\mathrm{C} 1=\mathrm{O} 1 \cdots \mathrm{H} 1 \mathrm{~A}$ & 2.656 & 59.4 & 83.8 & 111.3 \\
$\mathrm{C} 2=\mathrm{O} 2 \cdots \mathrm{H} 2 \mathrm{~A}$ & 2.874 & 63.6 & 72.7 & 110.7 \\
Favorable geometrics $^{33}$ & 2.72 & $50-60$ & 80 & 115
\end{tabular}



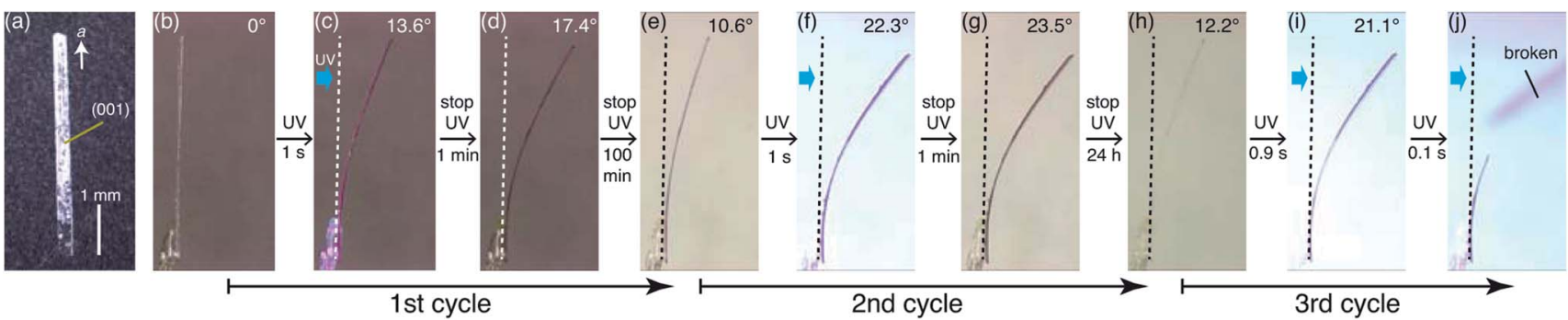

Fig. 5 First step photomechanical bending of a plate-like crystal of compound 1 upon UV light irradiation for $1 \mathrm{~s}$. (a) Top view of the plate-like crystal before light irradiation. $(b-j)$ Snapshots of side views of the plate-like crystal in $(b-d)$ the first stage of bending and relaxation, (e-f) the second stage of bending and relaxation, and $(\mathrm{h}-\mathrm{j})$ the third stage of bending. The angle at the upper right corner in each panel shows the tip displacement angle. Dotted lines indicate the initial position at the tip displacement angle $\left(0^{\circ}\right)$. UV radiation was always applied to the left side of the crystal.

\section{Bending behaviour}

First, bending of the plate-like crystal was observed after a short period of UV light irradiation (Fig. 5). From the X-ray diffraction analysis, the plate-like crystal was found to have a (001) face as its largest face, with a longitudinal direction along the $a$ axis (Fig. 5a). When the (001) face of the crystal (length: $4335 \mu \mathrm{m}$, width: $274 \mu \mathrm{m}$, thickness: $25 \mu \mathrm{m}$ ) was irradiated from the left by UV light ( $365 \mathrm{~nm}, 40 \mathrm{~mW} \mathrm{~cm}^{-2}$ ) for $1 \mathrm{~s}$, the crystal bent away from the light source up to the tip, with a displacement angle (see the angle definition in Fig. S5a $\dagger$ ) of $13.6^{\circ}$, and an accompanying crystal colour change from colourless to purple (Fig. 5b, c and Movie S1 $\dagger$ ). Gradually, the crystal bent up to $17.4^{\circ}$ even after the UV irradiation was stopped (Fig. 5d). Then the bending returned partially to $10.6^{\circ}$ (Fig. 5e) $100 \mathrm{~min}$ after the UV irradiation was stopped. The reasons that the crystal could not straighten back $100 \%$, can be due to the formation of the photoproducts via tri- $\pi$-methane and di- $\pi$-methane rearrangements, and the partial reverse of biradical 2 back to compound 1.

After a second application of UV radiation for $1 \mathrm{~s}$, the crystal bent up to $22.3^{\circ}$ (Fig. 5f). The crystal bent slightly to $23.5^{\circ}$ after the UV light was stopped (Fig. 5g). The bending returned gradually, reaching $12.2^{\circ}$ in $24 \mathrm{~h}$, with an accompanying colour change to colourless (Fig. 5h). Upon a third dose of UV exposure for $1 \mathrm{~s}$, the crystal bent to $21.1^{\circ}$, and broke into two pieces in the next moment (Fig. 5i and j). The temporal profile of the tip displacement angle is summarised in Fig. S5b. $\dagger$ The tip displacement angle at maximum bending decreased with the increase of crystal thickness (Fig. S6†).

A two-step bending motion was observed when another thin plate-like crystal (length: $525 \mu \mathrm{m}$, width: $134 \mu \mathrm{m}$, thickness: 8.6 $\mu \mathrm{m})$ was submitted to prolonged ( $1 \mathrm{~min}) \mathrm{UV}$ light irradiation (Fig. 6 and Movie S2†). In the first step, the crystal underwent bending upon initial UV light irradiation for $1 \mathrm{~s}$ (Fig. 6a and b). Under prolonged UV irradiation, the crystal returned to the straight form with decreasing purple colour after $9 \mathrm{~s}$ (Fig. 6c), and then bent up in the opposite direction with an additional colour change to light yellow after $50 \mathrm{~s}$ (Fig. 6d). The yellowcoloured bent crystal did not change its colour or shape after the UV irradiation was stopped, indicating that the second-step bending is irreversible.
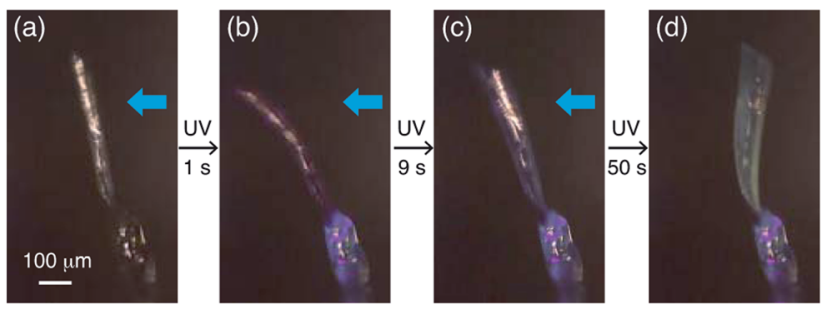

Fig. 6 Two-step bending of a plate-like crystal of compound 1 under continuous UV light exposure for $1 \mathrm{~min}$

\section{Bending mechanism}

The mechanism of the first-step bending is discussed based on the crystal structure change after short exposure to UV radiation (Table S1†). The initial X-ray diffraction measurement was performed on the crystal at $223 \mathrm{~K}$ before UV irradiation. Then, to clarify crystal structure changes due to biradical 2 , the crystal was irradiated for several seconds at $293 \mathrm{~K}$ while being rotated, because photochemical reactions are slow at low temperature. After a short period of UV irradiation, the crystallographic analysis was performed again at $223 \mathrm{~K}$, stabilising biradical 2 in the crystal.

The molecular arrangement of the (001) plane before light irradiation is shown in Fig. 4c. The carbonyl $\mathrm{C}=\mathrm{O}$ group is aligned almost parallel to the $a$ axis, which is the longitudinal direction of the crystal. After UV irradiation, biradical species 2 was not found, probably due to it not being present in sufficient quantities for structure determination, but the unit cell parameters changed slightly. The lengths of the $a$ and $b$ axes were elongated by $0.15 \%$ and $0.08 \%$, respectively, and the $c$ axis shortened by $0.19 \%$. This lattice change could have been induced by biradical species 2 , formed via an intramolecular $\gamma$ hydrogen abstraction, as reported by Hosoya and his coworker. ${ }^{34}$ They determined the crystal structure of compound $\mathbf{1}$ after UV light irradiation at low temperature (90 K), and detected an electron density peak of the methylene radical $\left(\cdot \mathrm{CH}_{2}^{-}\right)$as the disorder of the methyl group $\left(\mathrm{CH}_{3}\right)$ and the methylene radical. The abstracted hydrogen atom was also detected as a small electron density peak near the benzoyl oxygen atom with a distance of $0.967(2) \AA$, forming the benzyl-type radical. This 
result suggested that the $\gamma$-hydrogen atom transferred from the methyl group to the benzoyl oxygen atom, and that biradical 2 was produced during the hydrogen abstraction. Based on these results, biradical 2 might move away surrounding molecules along the $a$ axis due to hydrogen transfer to the carbonyl $\mathrm{C}=\mathrm{O}$, which is aligned almost parallel to the $a$ axis. This molecular change should induce the first-step bending due to elongation of the $a$ axis at the irradiated surface.

The second-step bending could have been induced by the formation of the photoproduct mixture via radical coupling of the biradical 2 , di- $\pi$-methane rearrangement, and tri- $\pi$ methane rearrangement (Fig. S3†). The bending speed and colour change observed during the second-step bending are in reasonably good agreement with the measured changes in UVvis and IR spectra.

\section{Conclusions}

A two-step bending motion of a plate-like crystal of compound 1 was observed under prolonged UV light irradiation. In the first step, the crystal bent away from the light source quickly upon initial light irradiation, with an accompanying colour change from colourless to purple. In the second step, under prolonged light irradiation, the crystal returned slowly to its straight form with decreasing purple colour, and then bent up in the opposite direction, accompanied by an additional colour change to light yellow. Based on the results of the spectroscopic measurements and X-ray crystallographic analysis, the first-step bending could have originated from the triplet biradical 2, generated by a Norrish type II intramolecular hydrogen abstraction. The subsequent second-step bending might have been induced by the photoproduct mixture via irreversible reactions under continuous UV exposure. Although the bending behaviour of crystals of compound $\mathbf{1}$ may not have practical applications, this work indicates that a typical photochemical reaction, namely, a Norrish type II intramolecular hydrogen abstraction, also induces photomechanical bending.

\section{Conflicts of interest}

There are no conflicts to declare.

\section{Acknowledgements}

This study was financially supported by the JSPS Grant-in-Aid for Scientific Research B (17H03107), Challenging Exploratory Research (16K12918), Research Fellowship for Young Scientists, and WISE project research (Research for Energy Next) at Waseda University. T. T. also would like to thank the Leading Graduate Program in Science and Engineering at Waseda University.

\section{References}

1 Y. Yu, M. Nakano and T. Ikeda, Nature, 2003, 425, 145.

2 Y. Takashima, S. Hatanaka, M. Otsubo, M. Nakahata,

T. Kakuta, A. Hashidzume, H. Yamaguchi and A. Harada, Nat. Commun., 2012, 3, 1270.
3 S. Kobatake, S. Takami, H. Muto, T. Ishikawa and M. Irie, Nature, 2007, 446, 778-781.

4 D. Kitagawa, H. Nishi and S. Kobatake, Angew. Chem., Int. Ed., 2013, 52, 9320-9322.

5 H. Koshima, N. Ojima and H. Uchimoto, J. Am. Chem. Soc., 2009, 131, 6890-6891.

6 T. Taniguchi, J. Fujisawa, M. Shiro, H. Koshima and T. Asahi, Chem.-Eur. J., 2016, 22, 7950-7958.

7 H. Koshima, K. Takechi, H. Uchimoto, M. Shiro and D. Hashizume, Chem. Commun., 2011, 47, 11423-11425.

8 R. Medishetty, A. Husain, Z. Bai, T. Runčevski, R. E. Dinnebier, P. Naumov and J. J. Vittal, Angew. Chem., Int. Ed., 2014, 53, 5907-5911.

9 R. Medishetty, S. C. Sahoo, C. E. Mulijanto, P. Naumov and J. J. Vittal, Chem. Mater., 2015, 27, 1821-1829.

10 R. O. Al-Kaysi and C. J. Bardeen, Adv. Mater., 2007, 19, 12761280.

11 L. Zhu, R. O. Al-Kaysi and C. J. Bardeen, J. Am. Chem. Soc., 2011, 133, 12569-12575.

12 H. Koshima, H. Uchimoto, T. Taniguchi, J. Nakamura, T. Asahi and T. Asahi, CrystEngComm, 2016, 18, 7305-7310.

13 New Frontiers in Photochromism, ed. M. Irie, Y. Yokoyama and T. Seki, Springer, Heidelberg, 2013, ch. 1 and 2.

14 M. Irie, T. Fukaminato, K. Matsuda and S. Kobatake, Chem. Rev., 2014, 114, 12174-12277.

15 P. Naumov, S. Chizhik, M. K. Panda, N. K. Nath and E. Boldyreva, Chem. Rev., 2015, 115, 12440-12490.

16 J. M. Abendroth, O. S. Bushuyev, P. S. Weiss and C. J. Barrett, ACS Nano, 2015, 9, 7746-7768.

17 T. Kim, L. Zhu, R. O. Al-Kaysi and C. J. Bardeen, ChemPhysChem, 2014, 15, 400-414.

18 P. Commins, I. T. Desta, D. P. Karothu, M. K. Panda and P. Naumov, Chem. Commun., 2016, 52, 13941-13954.

19 C. V. Kumar, B. A. R. C. Murty, S. Lahiri, E. Chackachery, J. C. Scaiano and M. V. George, J. Org. Chem., 1984, 49, 4923-4929.

20 C. V. Asokan, S. A. Kumar, S. Das, N. P. Rath and M. V. George, J. Org. Chem., 1991, 56, 5890-5893.

21 D. Ramaiah, M. C. Sajimon, J. Joseph and M. V. George, Chem. Soc. Rev., 2005, 34, 48-57.

22 P. R. Pokkuluri, J. R. Scheffer and J. Trotter, J. Am. Chem. Soc., 1990, 112, 3676-3677.

23 J. Chen, J. R. Scheffer and J. Trotter, Tetrahedron, 1992, 48, 3251-3274.

24 M. C. Sajimon, D. Ramaiah, C. H. Suresh, W. Adam, F. D. Lewis and M. V. George, J. Am. Chem. Soc., 2007, 129, 9439-9445.

25 J. J. Zhang and G. B. Schuster, J. Am. Chem. Soc., 1989, 111, 7149-7155.

26 G. M. Sheldrick, Acta Crystallogr., Sect. A: Found. Crystallogr., 2008, 64, 112-122.

27 CrystalStructure, version 4.2.2., Rigaku Corporation, Tokyo, Japan, 2016.

28 R. D. Small Jr and J. C. Scaiano, Chem. Phys. Lett., 1977, 50, 431-434.

29 L. J. Johnston and J. C. Scaiano, Chem. Rev., 1989, 89, 521547. 
30 H. Koshima, A. Maeda, N. Masuda, T. Matsuura, K. Hirotsu, K. Okada, H. Mizutani, Y. Ito, T. Y. Fu, J. R. Scheffer and J. Trotter, Tetrahedron: Asymmetry, 1994, 5, 1415-1418.

32 H. Koshima, M. Fukano, N. Ojima, K. Johmoto and H. Uekusa, J. Org. Chem., 2014, 79, 3088-3093.

33 H. Ihmels and J. R. Scheffer, Tetrahedron, 1999, 55, 885-907. 31 H. Koshima, D. Matsushige and M. Miyauchi, 34 S. Okabe and T. Hosoya, JPS Conf. Proc., $2015,8,031014$. CrystEngComm, 2001, 33, 1-3. 